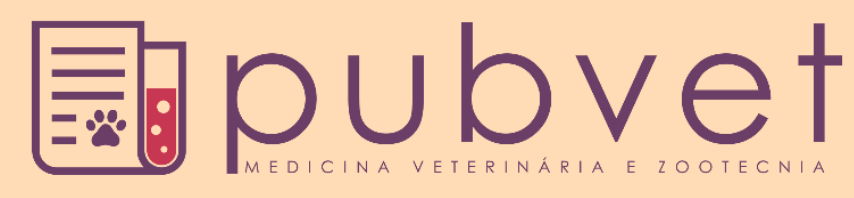

https://doi.org/10.31533/pubvet.v16n01a1001.1-9

\title{
Análise clínica de cães com parvovirose
}

\author{
Carollina Mariga ${ }^{1 *} \bullet$, Julia Carneiro Rodrigues Andrade ${ }^{1}$, Cinthia Melazzo de Andrade $^{2}$, \\ Alexandre Krause $^{2} \bullet$, Saulo Tadeu Lemos Pinto Filho ${ }^{2} \bullet$ \\ ${ }^{1}$ Graduanda de Medicina Veterinária na Universidade Federal de Santa Maria. Santa Maria - RS Brasil. \\ ${ }^{2}$ Professor da Universidade Federal de Santa Maria, Departamento de Clínica e Cirurgia de Pequenos Animais. Santa Maria - RS Brasil. \\ *Autor para correspondência, E-mail: carollina.mariga@hotmail.com
}

Resumo. Este estudo teve como objetivo classificar o perfil clínico dos cães acometidos por parvovirose no Hospital Veterinário Universitário da Universidade Federal de Santa Maria, localizado no Rio Grande do Sul. Para isso, foram revisados os históricos clínicos dos animais atendidos no hospital no período de janeiro de 2017 a julho de 2019, por meio dos prontuários dos pacientes. A prevalência foi de 44,3\% (78/176) para parvovirose. Os pacientes positivos foram classificados conforme raça, gênero, idade, estado de vacinação e, então, separados de acordo com os sistemas envolvidos: digestório, neuromuscular, multissistêmico, hematopoiético, respiratório, linfático, oftálmico, urinário e tegumentar, sinais clínicos e a presença de doenças concomitantes. A parvovirose ocorreu mais em machos de até um ano de idade e sem predisposição racial. Foi observado que a maioria dos cães não havia histórico de vacinação prévio. O presente estudo visa salientar a importância dessa infecção viral, que acomete principalmente animais jovens sem vacinação, reforçando que medidas de prevenção se tornam imprescindíveis para seu controle e melhor estado sanitário da população canina.

Palavras-chave: Canino, sinais clínicos, sistemas, parvovirose, perfil clínico

\section{Clinical analysis of dogs with parvovirus}

Abstract. This study aimed to classify the clinical profile of dogs affected by parvovirus at the Veterinary University Hospital of a higher education institution in Rio Grande do Sul. The clinical histories of the animals seen at the hospital from January 2017 to July 2019 were reviewed, using the patients' medical records. During this period, 176 animals were suspected to have parvovirus. The prevalence of disease was $44.3 \%$ (78/176). Positive patients were classified according to breed, gender, age, and vaccination status, then separated according to the bodily systems involved: digestive, neuromuscular, multisystemic, hematopoietic, respiratory, lymphatic, ophthalmic, urinary and cutaneous; clinical signs and the presence of concomitant diseases. Parvovirus occurs more often in males up to one year old and without predisposition for particular breeds. The researchers observed that most dogs had no previous vaccination history. This study highlights the infection patterns of this viral diseases, which mainly affects young unvaccinated animals, reinforcing the notion that preventive measures are essential for the control of the disease and the protection of the health of canine populations.

Key words: Canine, clinical signs, clinical profile, systems, parvovirus 


\section{Introdução}

A parvovirose se trata de uma das doenças infectocontagiosas de cães que possuem grande importância na clínica veterinária (Flores, 2012). O vírus da parvovirose canina (CPV-2) pertence à família Parvoviridae e teve seu surgimento a partir do vírus da panleucopenia felina (FPLV); porém, continuou sofrendo alterações genéticas, originando cepas denominadas CPV-2a e CPV-2b. No século XXI, houve o surgimento e disseminação mundial da cepa CPV-2c, considerando que esta cepa já foi isolada no Brasil (Streck et al., $\underline{2009)}$.

O parvovírus é altamente contagioso, com transmissão via oronasal por meio das fezes, fômites e ambientes contaminados. Por não possuir envelope, possui grande resistência no ambiente, sendo importante fonte de contaminação a outros animais (Flores, 2012). Após a infecção, o vírus possui tropismo por células de rápida multiplicação como células epiteliais intestinais, células precursoras da medula óssea e células do miocárdio (Goddard \& Leisewitz, 2010). As gastroenterites são comuns em cães jovens em todo o Brasil. Apesar de relatos de soro prevalência da parvovirose na Mesorregião Centro Ocidental RioGrandense, não há conhecimento do perfil clínico dos acometidos. É importante conhecer o perfil clínico da parvovirose adaptada à realidade de cada região, para que se possa estabelecer uma lista de diagnósticos diferenciais frente às diversas manifestações de uma doença, orientar o tutor na prevenção e facilitar o diagnóstico e tratamento precoce para obter maior taxa de sucesso e melhora clínica do paciente. Somado à identificação de fatores de risco, pode ser utilizado como indicador de prognóstico e estudos para planos para erradicação da enfermidade (Ogbu et al., 2021). Isto diminui os elevados níveis de mortalidade dos cães com parvovirose. Desta forma, o objetivo deste artigo é relatar o perfil clínico dos animais acometidos por parvovirose em um hospital veterinário universitário do Rio Grande do Sul.

\section{Materiais e métodos}

Foram revisados os históricos clínicos dos animais atendidos no Hospital Veterinário Universitário da Universidade Federal de Santa Maria (HVU-UFSM), Santa Maria/RS, no período de janeiro de 2017 a julho de 2019. Os cães com suspeita clínica de parvovirose foram testados para a presença do antígeno proteico da superfície do vírus, pelo teste imunocromatográfico rápido $\left(I D E X X^{\circledR}\right.$ e Alere $\left.^{\circledR}\right)$ por meio de swab retal ou amostras de fezes. Os pacientes positivos foram classificados conforme raça, gênero, idade, estado de vacinação e, então, separados de acordo com os sistemas envolvidos (digestório, neuromuscular, multissistêmico, hematopoiético, respiratório, linfático, oftálmico, urinário, tegumentar), sinais clínicos e doenças concomitantes. Quanto à idade, os pacientes foram divididos em três grupos de acordo com literatura: filhotes, até 1 ano de idade; adultos, de um a nove anos, e idosos, a partir dos dez anos de idade. Os valores hematológicos de referência são baseados em Schalm's (Weiss \& Wardrop, 2010), enquanto os valores bioquímicos são referentes ao Kaneko (Kaneko et al., 2008).

\section{Resultados}

Dos 11.910 caninos atendidos no HVU-UFSM no período de estudo, 176 (176/11.910, 1,5\%) foram suspeitos de parvovirose, destes, $78(44,3 \%)$ foram positivos no teste imunocromatográfico. Para o diagnóstico, em 75,6\% usou-se de swab retal, além de fezes $(17,9 \%)$ e plasma (1,3\%). Entre os pacientes positivos para parvovirose, 52,6\% (41/78) eram SRD e 47,4\% (37/78) eram de raças puras, sendo quatro Pastor Alemão, quatro Shih-tzu, quatro Border Collie e quatro Dachshund, três Boiadeiro-australiano, dois para cada das seguintes raças Labrador Retriever, Pequinês, Pug, Pinscher e Poodle e um representante para Buldogue, Rottweiler, Husky Siberiano, Rough Collie, Fila Brasileiro, Dogue Alemão, Boxer e Bull terrier. $61,5 \%$ (48/78) eram machos e 38,5\% (30/78), fêmeas. A faixa etária foi dividida em $53(67,9 \%)$ filhotes com menos de 6 meses de idade, 15 (19,2\%) filhotes entre 6 meses e um ano de idade, totalizando 87,1\% (68/78) filhotes. Entre os adultos, $9(11,5 \%)$ tinham mais de um ano e $1(1,3 \%)$ com mais de nove anos de idade (Figura 1).

A distribuição temporal de exames realizados em cães com suspeita de parvovirose foi de 46,0\% (81/176) em 2017, sendo 37 positivos e 44 negativos; 23,9\% (42/176) no ano de 2018, com 21 positivos e 21 
negativos e, 30,1\% (53/176) no ano de 2019, com 20 positivos e 30 animais negativos. Além disso, a distribuição de casos frente às estações do ano foi de 39,7\% (31/78) no verão, 26,9\% (21/78) durante o outono, 19,2\% (15/78) no inverno e 14,1\% (11/78) de positivos na primavera.

Sobre estado de imunização dos animais acometidos 44,8\% (35/78) não eram vacinados, 30,7\% (24/78) estavam durante o protocolo inicial de imunoprofilaxia e 16,6\% (13/78) estavam com a imunização em dia. Contudo, seis cães não possuíam esta informação no histórico. Daqueles em vacinação, 66,7\% tinham apenas a primeira dose e $25,0 \%$ haviam recebido o primeiro reforço.

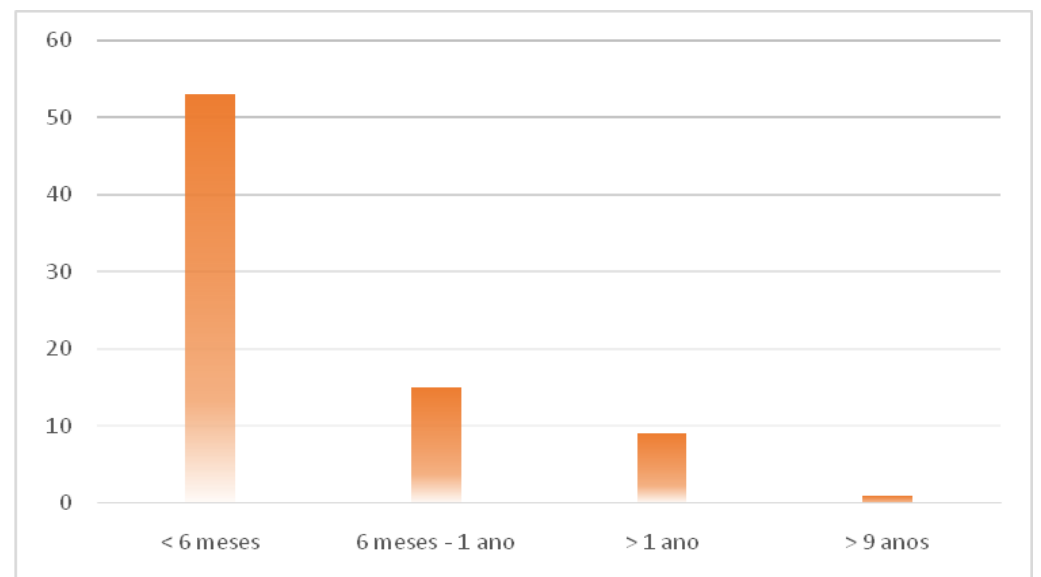

Figura 1. Distribuição etária dos animais positivos para parvovirose no HVU-UFSM de janeiro de 2017 a julho de 2019 em números absolutos

Dos 22 animais com informações específicas sobre o tipo de vacina, 63,6\% (14/22) receberam imunizante produzido no Brasil e 36,4\% (8/22), importado. A prevalência da parvovirose desta pesquisa foi de 47,4\% (37/78) em 2017, 26,9\% (21/78) de 2018 e 25,6\% (20/78) até julho de 2019.

Setenta e dois animais $(92,3 \%)$ apresentavam sinais clínicos relacionados com a parvovirose. Os 7,7\% restantes realizaram o exame imunocromatográfico, mas sem justificativa na ficha de atendimento, o que torna impossível inferir se apresentavam sinais clínicos característicos.

O sistema mais acometido foi o digestório, com 98,6\% (71/72) de presença nos casos relatados. Os sinais clínicos mais prevalentes foram êmese com 86,1\% (62/72) de representatividade, sendo 9,7\% (6/62) com a presença de sangue; diarreia em 76,4\% (55/72) dos casos - destes, 41,8\% (23/55) com presença de sangue vivo, 5,4\% (3/55) com melena e 52,7\% (29/55) sem sinais de hemorragia intestinal, e desidratação presente em 50\% dos casos. Os demais sinais clínicos acometidos estão listados na tabela 1.

Tabela 1. Prevalência dos sinais clínicos do sistema digestório dos animais positivos para parvovirose de janeiro 2017 a julho de 2019

\begin{tabular}{lcc}
\hline Sinais clínicos & Número absoluto (x/72) & Número relativo $(\%)$ \\
\hline Êmese & 62 & 86,1 \\
Diarreia & 55 & 76,4 \\
Desidratação & 36 & 50,0 \\
Emagrecimento & 10 & 13,9 \\
Sialorreia & 7 & 9,7 \\
Melena & 6 & 8,3 \\
Adipsia & 5 & 6,9 \\
Hiporexia & 3 & 4,2 \\
Inapetência & 3 & 4,2 \\
Caquexia & 2 & 2,8 \\
Dor a palpação abdominal & 2 & 2,8 \\
Hematoquezia & 2 & 2,8 \\
Oligodipsia & 2 & 2,8 \\
Polidipsia & 2 & 2,8 \\
Prolapso retal & 1 & 1,4
\end{tabular}


Um cão apresentou endoparasitas nas fezes. Também houve relato de animal com aumento de volume abdominal. O segundo sistema mais acometido nos pacientes positivos para parvovirose canina foi o neuromuscular com $61,1 \%$ (44/72) dos casos. Apatia foi o sinal clínico mais presente com 56,9\% (41/72). Os demais sinais clínicos relatados estão na tabela 2.

Tabela 2. Prevalência dos sinais clínicos do sistema neuromuscular dos animais positivos para parvovirose de janeiro 2017 a julho de 2019

\begin{tabular}{lcc}
\hline Sinais clínicos & Número absoluto (x/72) & Número relativo (\%) \\
\hline Apatia & 41 & 56,7 \\
Obnubilação & 4 & 5,6 \\
Fraqueza & 3 & 4,2 \\
Estado semicomatoso & 1 & 1,4 \\
Tremores & 1 & 1,4 \\
\hline
\end{tabular}

Os demais sistemas e sinais clínicos relatados estão listados na tabela 3.

Tabela 3. Prevalência dos sinais clínicos distintos dos sistemas gastrointestinal e neuromuscular relacionados aos animais positivos para parvovirose de janeiro de 2017 a julho de 2019

\begin{tabular}{|c|c|c|c|}
\hline Sistemas & Sinal Clínico & Número absoluto (x/72) & Número relativo, $\%$ \\
\hline \multirow{4}{*}{ Multissistêmico } & Hipertermia & 9 & 12,5 \\
\hline & Hipotermia & 7 & 9,7 \\
\hline & Hiperglicemia & 3 & 4,2 \\
\hline & Hipoglicemia & 1 & 1,4 \\
\hline \multirow{2}{*}{ Hematopoiético } & Mucosas pálidas & 15 & 20,8 \\
\hline & Hipotenso & 1 & 1,4 \\
\hline \multirow{4}{*}{ Respiratório } & Secreção nasal & 2 & 2,8 \\
\hline & Dispneia & 1 & 1,4 \\
\hline & Taquipneia & 1 & 1,4 \\
\hline & Tosse & 1 & 1,4 \\
\hline Linfático & Linfadenomegalia & 5 & 6,9 \\
\hline Oftálmico & Secreção ocular & 4 & 5,5 \\
\hline \multirow{2}{*}{ Urinário } & Hematúria & 1 & 1,4 \\
\hline & Urina escura & 1 & 1,4 \\
\hline \multirow{6}{*}{ Tegumentar } & Atopia & 1 & 1,4 \\
\hline & Dermatite & 1 & 1,4 \\
\hline & Lesão cervical & 1 & 1,4 \\
\hline & Otite & 1 & 1,4 \\
\hline & Prurido & 1 & 1,4 \\
\hline & Pústulas abdominais & 1 & 1,4 \\
\hline
\end{tabular}

Em todos os animais que apresentaram linfadenomegalia o linfonodo afetado era o mandibular. Na parvovirose, dois animais foram a óbito durante o atendimento $(2,8 \%)$ e três $(4,2 \%)$ apresentaram sinais após início da vacinação.

Cinquenta e quatro $(75,0 \%)$ dos 72 animais positivos sintomáticos realizaram exames hematológicos. No eritrograma, 38,9\% (21/54) apresentaram anemia, 8 (14,8\%) tinham hemograma compatível com policitemia (aumento de células vermelhas e proteínas plasmáticas). Em cinco (9,3\%) animais identificouse baixos níveis de proteína plasmática e dez (18,5\%), hipoalbuminemia. Dez $(18,5 \%)$ manifestaram trombocitopenia, enquanto cinco $(9,3 \%)$, trombocitose. Trinta e sete $(68,5 \%)$ animais estavam leucopênicos, $22(40,7 \%)$ com diferenciação leucocitária por neutropenia; 19 (35,2\%), linfopenia; 17 (31,5\%), eosinopenia e $7(13,0 \%)$ com monocitopenia. Apenas um $(1,8 \%)$ animal teve leucocitose por neutrofilia, mas houve 5 $(9,3 \%)$ registros de monocitose e $2(3,7 \%)$ de neutrofilia sem associação com leucocitose. O bioquímico revelou hiperalbuminemia em $2(3,7 \%)$ animais.

\section{Discussão}

A apresentação clínica da parvovirose se dá por meio de duas síndromes: a gastroentérica, comum em animais jovens e caracterizada pela necrose das vilosidades intestinais, resultando em diarreia, que pela má 
absorção e o aumento de permeabilidade da mucosa geralmente há a presença de sangue, mas há relatos de casos em que pode não estar presente (Flores, 2012). No trabalho aqui realizado a forma gastroentérica foi a mais descrita já que estes sinais estavam presentes em 98,6\% dos casos. Necropsias realizadas entre 19652004 no LPV-UFSM confirmaram 7,2\% morte por causa da parvovirose, entretanto, foi a doença mais diagnosticada em filhotes $(18,4 \%)$ (Fighera et al., 2008).

A clínica da parvovirose é caracterizada por sinais como vômito e diarreia em associação com anorexia, depressão e febre. Há severa desidratação e possível choque hipovolêmico pela perda de fluidos e proteínas pelas lesões através do trato gastrointestinal (Franzo et al., 2019). No presente estudo muitos animais foram acometidos por vômito, diarreia, apatia e desidratação. Alterações no estado de consciência dos pacientes também foram relatadas, como o estado comatoso, que pode ser observado no choque hipovolêmico ou choque séptico. A presença de sangue nas fezes também tem sido sugestiva como consequência da endotoxemia e produção de certas citocinas e não somente às lesões no epitélio intestinal causada pelo vírus (Goddard \& Leisewitz, 2010).

A soro prevalência de parvovírus na cidade de Santa Maria/RS foi de 68,7\%, confirmando que há disseminação e prevalência do CPV na população, principalmente pela sua alta resistência ambiental (Dezengrini et al., 2007). Contudo, a positividade deste estudo foi inferior se comparada à soropositividade apresentada acima e da soropositividade de 96,2\% em Passo Fundo/RS (Strottmann et al., 2008). Apesar do teste utilizado neste trabalho detectar antígeno e garantir cerca de $98 \%$ de especificidade e $95 \%$ de sensibilidade, há possibilidades de resultados falsos negativos, por isso, deve-se recorrer a outros métodos de diagnóstico, como hemaglutinação, ensaio imunoenzimático e reação em cadeia da polimerase (PCR). Um estudo ainda destaca baixa sensibilidade de detecção de antígeno e altos títulos de anticorpos contra CPV-2 no lúmen intestinal, resultando que os anticorpos da mucosa bloqueiam as partículas virais, o que pode incorrer em falsos negativos em testes imunocromatográficos, mesmo nos casos clínicos de parvovirose (Decaro \& Buonavoglia, 2012).

Uma pesquisa considerou idade, raça, gênero, localização geográfica, vacinação e estado sanitário como fatores de risco para parvovirose, associados com estresse (desmame precoce e alta carga parasitária), imunidade insuficiente, região geográfica e a presença de doenças concomitantes para o desenvolvimento dos sinais clínicos (Ogbu et al., 2021). A parvovirose pode afetar cães de qualquer idade, sendo mais comum entre seis semanas e quatro meses de idade. Outros autores estendem essa suscetibilidade até os sete meses de idade (Nandi \& Kumar, 2010). Nos nossos achados, os filhotes foram mais significativamente afetados, cerca de $86,1 \%$ dos casos em animais de até um ano de idade, o que corrobora os dados supracitados, enquanto $11,4 \%$ dos animais já eram considerados adultos (acima de um ano). Apesar da doença acometer diversas idades, a severidade é maior em pacientes com menos de três meses de idade, evidenciando a importância do cuidado nesta fase, já que no período de dois a quatro meses houve a maior concentração dos filhotes acometidos. Fatores que predispõem à infecção em filhotes são questões imunológicas, parasitas intestinais, superlotação em canis, locais pouco higiênicos e estresse por condições ambientais (Ogbu et al., 2021). Outro estudo afirma que comorbidades como coronavírus e parasitas intestinais podem influenciar na eficácia da vacina já que causam anemia, fraqueza, estresse e infecções bacterianas secundárias (Cunha et al., 2020). A presença da enfermidade em dois animais considerados idosos é considerada incomum (Nandi \& Kumar, 2010). Esses relatos incomuns podem ser justificados devido às mudanças do cenário global, aumento das taxas de mutação do vírus resultando em circulação de novas cepas, aumento da resistência no ambiente, ausência de reforço vacinal e imunocomprometimento senil. Estudos relataram uma incidência $16,7 \%$ de CPV-2c em animais adultos, especula ser relacionado à falta de imunidade, por vacinação com antígenos apenas para CPV-2a ou $2 b$, ou por exposição natural às outras cepas de parvovírus (Oliveira et al., 2018). No presente estudo não houve sequenciamento genético para checagem de cepas, em contrapartida, estudos afirmam que as vacinas atuais conferem imunidade contra as cepas CPV-2, CPV-2b e CPV-2c (Mazzaferro, 2020).

A suscetibilidade à parvovirose em certas raças ainda não é bem definida, mas algumas raças como Rottweiler, Doberman pinscher, American Pit Bull terrier, Labrador Retriever e Pastor Alemão parecem ser 
mais suscetíveis que outras (Goddard \& Leisewitz, 2010). Neste estudo houve maior incidência entre os animais de raças definidas como Pastor Alemão, Shih-tzu, Border Collie, Dachshund e Boiadeiroaustraliano. Relevam-se os machos inteiros porque apresentam o dobro de risco para desenvolver a doença, enquanto neste artigo, apesar de maior incidência nos machos, a diferença $(60,8 \%$ contra $39,2 \%)$ não é tão acentuada (Goddard \& Leisewitz, 2010).

Relatos mostram que a parvovirose apresenta-se com discreta sazonalidade, possuindo pico de ocorrência nos meses de verão, enquanto há queda nos meses de inverno (Goddard \& Leisewitz, 2010). Outros relatos afirmam sazonalidade com pico entre fim da primavera e início do verão, enquanto as taxas são menores do início do outono ao fim do inverno (Horecka et al., 2020). No atual estudo, a incidência foi semelhante nos meses de verão e outono, enquanto a queda se deu nos meses de primavera. Provavelmente esta diferença se deu pela ausência de casos na primavera de 2018 e o maior número de casos do levantamento registrados no outono de 2019. Ressalta-se que quase metade dos casos foram registrados no ano de 2017, enquanto 2018 e 2019 apresentaram semelhante número de casos, mesmo com 2019 não sendo contemplado inteiramente no levantamento. A presença de endoparasitas já foi associado como fator que pode predispor ou agravar a parvovirose, em contrapartida não se pode relacionar a presença do parasitismo com a severidade da doença. Neste estudo, apenas um animal apresentou parasitas visivelmente nas fezes, porém, infelizmente, o coproparasitológico não é um exame frequentemente solicitado na rotina analisada (Castro et al., 2007). Portanto, é possível que mais cães estivessem parasitados, mas sem o devido diagnóstico. $\mathrm{O}$ aumento de volume abdominal relatado em um dos cães pode estar relacionado com parasitismo intestinal e consequente formação de transudato, principalmente em filhotes (Rodrigues \& Molinari, 2018). Estudos afirmam que parasitas intestinais aumentam a morbidade e a mortalidade da doença (Mazzaferro, 2020). Enquanto outros associaram a gravidade de uma enterite apresentada por um filhote com parvovirose com a infecção por Cryptosporidium devido à alta taxa de renovação celular e consequente taxa de replicação viral e destruição de enterócitos (Denholm et al., 2001). Cita-se uma enterite menos grave causada pelo coronavírus, doença com baixa letalidade. Também ressalta verminose por Ancylostoma caninum, acometendo filhotes com diarreia sanguinolenta e ainda relata isosporose, doença que acomete animais jovens em quadro febril e diarreia mucoide com eventuais estrias de sangue (Megid et al., 2016). A enterite causada pela cepa CPV-2c é mais mucoide se comparada às outras cepas do parvovírus (Desario et al., 2005); porém, nenhuma das doenças acima comparadas causam leucopenia e imunossupressão.

Os sinais gastrointestinais decorrentes da infecção por CPV-2 são acompanhados de imunossupressão severa, caracterizada por linfopenia podendo apresentar também pancitopenia (Dezengrini et al., 2007). Dos 54 animais que realizaram exame de sangue, $37(68,5 \%)$ apresentaram leucopenia sendo, principalmente por neutropenia $(59,5 \%)$, linfopenia $(51,3 \%)$ e eosinopenia $(45,9 \%)$. Em estudo realizado revelou que 26 cães, entre 39 positivos para parvovirose $(66,7 \%)$, apresentaram leucopenia (Simon et al., 2019), enquanto outro estudo a caracteriza como leucopenia por neutropenia e linfopenia (Biezus et al., 2018). Estudos mostram que animais com linfopenia e hipoalbuminemia no momento de internação têm associação com o tempo de hospitalização necessário para recuperação (Mazzaferro, 2020).

A porcentagem dos leucopênicos se deu acima dos $100 \%$ devido aos diferentes perfis, que podem ser resultados de mais de um tipo de célula. A anemia é comum na parvovirose como consequência da predileção e injúria na medula óssea e devido à perda sanguínea intestinal (Simon et al., 2019; Weiss et al., 1999) e foi a principal alteração encontrada neste artigo, confirmada laboratorialmente em 28,9\% (21/54) dos cães. Clinicamente, 20,8\% (15/72) apresentavam palidez de mucosas que é indicativo desta alteração.

Casos de parvovirose em cães durante o período de vacinação já tinha sido relatado e indicam janela de suscetibilidade dos filhotes, consequência dos baixos níveis de anticorpos maternos e sistema imunológico imaturo, ainda não capaz de responder efetivamente à imunização (Fontana et al., 2013), considerado o principal fator de risco para falha vacinal (Monteiro et al., 2016). Neste caso, 30,7\% estavam durante o período inicial de vacinação. Destes, $66,7 \%$ estavam apenas com uma dose e $25,0 \%$ já haviam recebido com duas doses. Em artigo com filhotes positivos para parvovirose, 54,0\% possuíam apenas uma dose do 
imunizante e 35,1\% apresentavam duas doses, enquanto apenas 13,5\% possuíam imunização em dia. Entre os animais de uma pesquisa, 52,4\% possuíam apenas a primeira dose, enquanto 47,6\% receberam as duas, concluindo que não houve diferença significativa de prevalência da doença entre animais vacinados ou não (Ogbu et al., 2021). Segundo estudo realizado no estado de São Paulo, cães vacinados e acometidos pela parvovirose possuem maior taxa de óbito se comparada com os doentes que não foram vacinados. Os autores sugerem que diferenças na mortalidade podem estar relacionadas à demora dos tutorem em levar os cães para realizarem a terapia no hospital onde a pesquisa foi realizada. As falhas dos tutores em completar o protocolo vacinal, muitas vezes administrando apenas uma ou duas doses do imunizante, também pode ter contribuído para este resultado (Monteiro et al., 2016). Contudo, avaliar a taxa de óbitos entre imunizados e não imunizados não foi o escopo da nossa pesquisa, uma vez que pacientes com diagnóstico positivo para parvovirose no HVU-UFSM são encaminhados para outras clínicas do município, o que dificulta o descobrimento do desfecho dos casos.

As novas mutações genotípicas do vírus da parvovirose (CPV-2a, 2b e 2c) influenciam na eficácia das vacinas há mais tempo no mercado e consequente falha na proteção dos animais vacinados contra a doença (Castro et al., 2011). Um estudo revelou que a maioria dos animais vacinados $(85,71 \%)$ estava infectada pela CPV-2c, sendo a cepa predominante entre animais vacinados e não vacinados com parvovírus (Yip et al., 2020). No nosso estudo, apenas 37 animais tinham informações sobre a origem e tipo de imunizante, contabilizando $37,8 \%$ (14/37) da nacional e 21,6\% (8/37) da importada, mas não foi possível realizar a análise de qual cepa foi a infectante nos participantes. Nos registros dos outros cães apenas era informado o tipo de vacina e não sua origem, dado relevante pela presença de diferentes cepas e possível associação com as falhas vacinais (Castro et al., 2011). São considerados erros vacinais: influência de imunidade materna, imunidade não responsiva à vacinação, reversão para virulência, erros de manipulação e estocagem das vacinas e cepas variantes do CPV (Decaro et al., 2020). A circulação dos vírus da cinomose canina e do parvovírus entre a população canina da cidade de Santa Maria/RS torna importante a prevenção da doença principalmente pela correta imunização, por isso são necessários estudos para reconhecer a prevalência e a circulação das diferentes cepas da região (Dezengrini et al., 2007).

\section{Conclusão}

Os resultados deste estudo permitem concluir que a prevalência da parvovirose foi de 44,9\%. Com maior positividade em machos, SRD e filhotes, seguido dos adultos e com pontuais relatos em idosos. O estado sanitário foi de $44,8 \%$ de não vacinados dentre os animais que testaram positivo e participaram da pesquisa. O sistema de maior acometimento foi o digestório, com sinais clínicos de vômito e diarreia.

\section{Conflito de interesse}

Declaramos que não possuímos nenhum conflito de interesse.

\section{Referências bibliográficas}

Biezus, G., Casagrande, R. A., Ferian, P. E., Luciani, M. G., Souza, J. R., Cristo, T. G., Dal Pozo, S., \& Vargas, C. B. (2018). Ocorrência de parvovirose e cinomose em cães no Planalto Catarinense. Revista de Ciências Agroveterinárias, 17(3), 396-401.

Castro, T X, Costa, E. M., Leite, J. P., Labarthe, N. V, \& Garcia, R. C. N. (2011). Monitoring of canine parvovirus (CPV) strains detected in vaccinated puppies in Brazil. Research in Veterinary Science, 90(2), 336-340.

Castro, Tatiana Xavier, Uchoa, C. M. A., Albuquerque, M. C., Labarthe, N. V., \& Garcia, C. N. C. (2007). Canine parvovirus (CPV) and intestinal parasites: laboratorial diagnosis and clinical signs from puppies with gastroenteritis. International Journal Applled Research Medicine, 5(2), 72.

Cunha, R. D. S., Silva Junior, C. L., Costa, C. A., Aguiar, H. M., \& Junqueira Júnior, D. G. (2020). Comparison of immunity against canine distemper, adenovirus and parvovirus after vaccination with 
two multivalent canine vaccines. Veterinary Medicine and Science, 6(3), 330-334. https://doi.org/10.1002/vms3.274.

Decaro, N, Buonavoglia, C., \& Barrs, V. R. (2020). Canine parvovirus vaccination and immunisation failures: Are we far from disease eradication? Veterinary Microbiology, 247, 108760.

Decaro, Nicola, \& Buonavoglia, C. (2012). Canine parvovirus - a review of epidemiological and diagnostic aspects, with emphasis on type 2c. Veterinary Microbiology, 155(1), 1-12. https://doi.org/10.1016/j.vetmic.2011.09.007.

Denholm, K. M., Haitjema, H., Gwynne, B. J., Morgan, U. M., \& Irwin, P. J. (2001). Concurrent Cryptosporidium and parvovirus infections in a puppy. Australian Veterinary Journal, 79(2), 98-101.

Desario, C., Decaro, N., Campolo, M., Cavalli, A., Cirone, F., Elia, G., Martella, V., Lorusso, E., Camero, M., \& Buonavoglia, C. (2005). Canine parvovirus infection: which diagnostic test for virus? Journal of Virological Methods, 126(1-2), 179-185. https://doi.org/10.1016/j.jviromet.2005.02.006.

Dezengrini, R., Weiblen, R., \& Flores, E. F. (2007). Soroprevalência das infecções por parvovírus, adenovírus, coronavírus canino e pelo vírus da cinomose em cães de Santa Maria, Rio Grande do Sul, Brasil. Ciência Rural, 37(1), 183-189.

Fighera, R. A., Souza, T. M., Silva, M. C., Brum, J. S., Graça, D. L., Kommers, G. D., Irigoyen, L. F., \& Barros, C. S. L. (2008). Causas de morte e razões para eutanásia de cães da Mesorregião do Centro Ocidental Rio-Grandense (1965-2004). Pesquisa Veterinária Brasileira, 28(4), 223-230. https://doi.org/10.1590/s0100-736x2008000400005.

Flores, E. F. (2012). Virologia veterinária. Universidade Federal de Santa Maria.

Fontana, D. S., Rocha, P. R. D., Cruz, R. A. S., Lopes, L. L., Melo, A. L. T., Silveira, M. M., Aguiar, D. M., \& Pescador, C. A. (2013). A phylogenetic study of canine parvovirus type $2 \mathrm{c}$ in midwestern Brazil. Pesquisa Veterinária Brasileira, 33, 214-218.

Franzo, G., Tucciarone, C. M., Casagrande, S., Caldin, M., Cortey, M., Furlanello, T., Legnardi, M., Cecchinato, M., \& Drigo, M. (2019). Canine parvovirus (CPV) phylogeny is associated with disease severity. Scientific Reports, 9(1), 1-8. https://doi.org/10.1038/s41598-019-47773-6.

Goddard, A., \& Leisewitz, L. (2010). Canine parvovirus. Veterinary Clininic North American. Small Animal Practice, 40, 1041-1053.

Horecka, K., Porter, S., Amirian, E. S., \& Jefferson, E. (2020). A decade of treatment of canine parvovirus in an animal shelter: A retrospective study. Animals, 10(6), 939.

Kaneko, J. J., Harvey, J. W., \& Bruss, M. L. (2008). Clinical biochemistry of domestic animals (6th ed. (ed.); Vol. 1). Academic press.

Mazzaferro, E. M. (2020). Update on canine parvoviral enteritis. Veterinary Clinics: Small Animal Practice, 50(6), 1307-1325.

Megid, J., Ribeiro, M. G., \& Paes, A. C. (2016). Doenças infecciosas em animais de produção e de companhia. Roca.

Monteiro, K., Allendorf, S. D., Vicente, A. F., Appolinário, C. M., Peres, M. G., Cortez, A., Heinemann, M. B., \& Megid, J. (2016). Viral type characterization and clinical aspects of canine parvovirus in naturally infected dogs in São Paulo State, Brazil. Pesquisa Veterinária Brasileira, 36, 1181-1185.

Nandi, S., \& Kumar, M. (2010). Canine parvovirus: current perspective. Indian Journal of Virology, 21(1), 31-44.

Ogbu, K. I., Chukwudi, I. C., Mira, F., Eze, U. U., Di Bella, S., Olaolu, O. S., Tion, M. T., Purpari, G., Cannella, V., \& Nwosuh, I. C. (2021). Current status and risk factors of canine parvovirus type 2 in North Central Nigeria. Comparative Immunology, Microbiology and Infectious Diseases, 74, 101578. https://doi.org/10.1016/j.cimid.2020.101578.

Oliveira, P. S. B., Cargnelutti, J. F., Masuda, E. K., Fighera, R. A., Kommers, G. D., Silva, M. C. da, Weiblen, R., \& Flores, E. F. (2018). Epidemiological, clinical and pathological features of canine 
parvovirus 2c infection in dogs from southern Brazil. Pesquisa Veterinária Brasileira, 38, 113-118. https://doi.org/10.1590/1678-5150-pvb-5122.

Rodrigues, B., \& Molinari, B. L. D. (2018). Diagnóstico e tratamento de parvovirose canina: Revisão de literatura. Brazilian Journal of Surgery and Clinical Research, 21(2), 127-134.

Simon, A. B., Nogueira, K. B. I., Ramos, D. G. S., \& Braga, Í. A. (2019). Análise hematológica de cães reagentes ao teste imunocromatográfico para parvovírus canino. Revista Interação Interdisciplinar, 3(1), $16-36$.

Streck, A. F., Souza, C. K., Gonçalves, K. R., Zang, L., Pinto, L. D., \& Canal, C. W. (2009). First detection of canine parvovirus type 2c in Brazil. Brazilian Journal of Microbiology, 40(3), 465-469. https://doi.org/10.1016/j.vetmic.2007.04.028.

Strottmann, D. M., Scortegagna, G., Kreutz, L. C., Barcellos, L. J. G., Frandoloso, R., \& Anziliero, D. (2008). Diagnóstico e estudo sorológico da infecção pelo parvovírus canino em cães de Passo Fundo, Rio Grande do Sul, Brazil. Ciência Rural, 38(2), 400-405.

Weiss, D J, \& Wardrop, J. K. (2010). Schalm's Veterinary Hematology.

Weiss, Douglas J, Evanson, O. A., \& BVSc, J. S. (1999). A retrospective study of canine pancytopenia. Veterinary Clinical Pathology, 28(3), 83-88.

Yip, H. Y. E., Peaston, A., Woolford, L., Khuu, S. J., Wallace, G., Kumar, R. S., Patel, K., Ahani Azari, A., Akbarzadeh, M., \& Sharifian, M. (2020). Diagnostic challenges in canine parvovirus 2c in vaccine failure cases. Viruses, 12(9), 980.

Histórico do artigo:

Recebido: 14 de agosto de 2021

Aprovado: 14 de setembro de 2021

Disponível online: 4 de novembro de 2021
Licenciamento: Este artigo é publicado na modalidade Acesso Aberto sob a licença Creative Commons Atribuição 4.0 (CC-BY 4.0), a qual permite uso irrestrito, distribuição, reprodução em qualquer meio, desde que $o$ autor e a fonte sejam devidamente creditados 